\title{
Experimental validation of an anisotropic delay damage model for impact on reinforced concrete structures
}

\author{
M. Chambart (1,2), F. Gatuingt (1), R. Desmorat (1) and D. Guilbaud (2) \\ (1) LMT-Cachan, ENS Cachan/UPMC/CNRS/PRES UniverSud Paris, France \\ (2) DEN/DM2S/SEMT, CEA Saclay, 91191 Gif-sur-Yvette cedex, France
}

Impact problems on reinforced concrete structures are usually computed with models coupling plasticity and isotropic damage. The induced damage anisotropy observed for quasi-brittle materials such as concrete is often reproduced considering different variables for tension and compression (not consistent with the thermodynamic framework). Introducing viscosity for both damage and plasticity evolutions enables to reproduce the strength enhancement due to rate effects. Such kinds of models present the main advantage to describe precisely each phenomenon locally observed (different rate effects in traction and compression, compaction under confined loadings ...) but require a large number of parameters. Anisotropic damage is quite relevant to describe the micro-cracking pattern and the failure conditions of quasi-brittle materials and structures. In concrete, a state of micro-cracks orthogonal to the loading direction in tension and parallel to it in compression is easily described by a second order damage variable. This anisotropic delay-damage model, used in this work, introduces only few parameters ( 7 including elasticity parameters $E$ and $\nu$ ) compared to the ones mentioned higher. The efficiency and the validation of such an approach is illustrated with its application on impacted reinforced concrete beams and dynamic Brazilian tests. The test has been performed with the drop-weight tower ORION of the CEA Saclay for two kinds of beam geometries in order to exhibit flexion and shear rupture.

\section{INTRODUCTION}

The challenge of understanding the behavior of civil engineering structures under dynamic loading is usually linked to security issues but also some economic problems. Advances in instrumentation continue to improve our understanding on these subjects, so one continues around the world to develop impact tests on reinforced concrete structures. The civil engineering is one area where the tests are the most numerous. Indeed, it's only repeating the experiments and varying the parameters that we managed to understand the behavior of structures. But few cases where experiments are conducted on real structures with the stresses actually envisaged, are available. Moreover these experiments do not always provide more information than mockup models. Therefore, we find in the literature a large number of tests on simple structures such as slabs or beams. The philosophy of these kind of tests is to demonstrate on simple structures (beams, slabs) a number of local and global phenomena to allow modeling of more complex cases. Tests developed in the Dynamics Laboratory (DYN) of the CEA Saclay during this work include in this perspective. Two types of tests on a drop-weight tower were made: dynamic Brazilian tests and impact tests on beams. The dynamic Brazilian tests are relatively uncommon in the literature ( (Tedesco, Ross, and Kuennen 1993) made the first dynamic Brazilian tests on split Hopkinson bars), but very interesting. A fast digital camera has been used in addition to more traditional, but precise and efficient, instrumentations (accelerometer, displacement measurement by camera, force sensors). The recorded images are analyzed by Digital Image Correlation with the software CORRELI developed at the LMT Cachan (Besnard, Hild, and Roux 2006). The impact tests on beams were designed to study the transition from a ductile failure mode (flexural) to a brittle fracture mode (shear cone). The two factors influencing this study are the slenderness of the beam and the transverse reinforcement (stirrups). An anisotropic delay-damage model has been developed during this work and it is presented in the last section. Finally this model is used to model the tests realized on the drop-weight tower. 


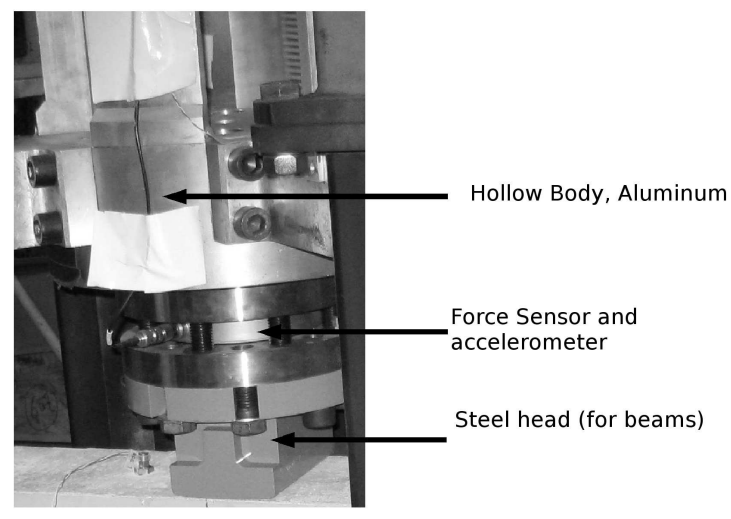

(a) Projectile and its instrumentation.

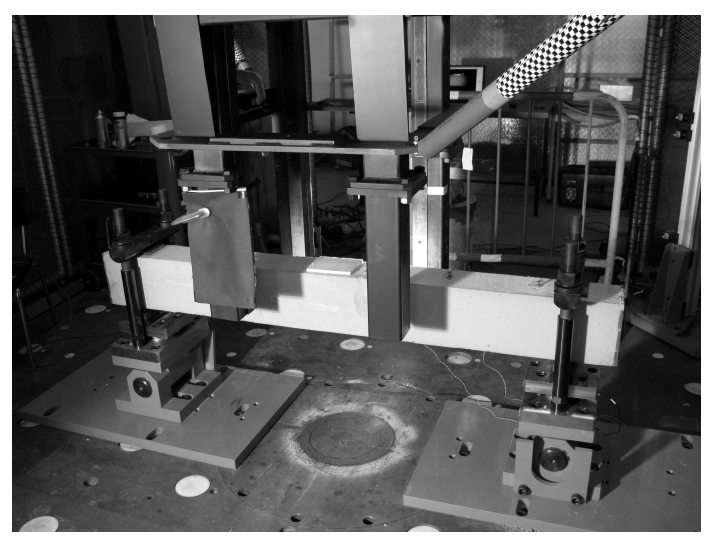

(b) Set-up for impact on beam

Figure 1: Experimental set-up for impact test on reinforced concrete beams

\section{IMPACT TESTS ON A DROP-WEIGHT TOWER}

Dynamic experiments on concrete can be classified into two classes, the ones aiming at characterizing the dynamic behavior of the material itself and the one focusing on the global structural response. In both cases a long tradition exists, and the technical improvements are perpetual. The experiments presented in this paper are widely based on the background in this domain. They consist in impact tests on a drop-weigth tower, first on plain concrete cylindrical specimens (brazilian or splitting test) then on reinforced concrete beams. These experiment thus cover the two fields of dynamical tests. The novelty is here the use of a high speed digital video camera associated with images correlation for the analysis of displacements fields.

\subsection{Experimental set-up}

The drop-weight tower Orion enables to drop a projectile up to a heigth of $7.00 \mathrm{~m}$. The projectile is constituted of a hollow body in aluminum and of a interchangeable head in steel, whose total mass worthes $100 \mathrm{~kg}$. For dynamical brazilian tests, we use a flat circular head whose diameter is $22 \mathrm{~cm}$, as the specimen length. For impact on beams, the head in rectangular $\left(11 * 20 \mathrm{~cm}^{2}\right)$ and slightly spherical. A force sensor is located between the projectile head and its body to record the impact force. An accelerometer is also placed there in order to correct the force measured since it also takes into account the inertial forces due to the projectile mass.

By integration of the signal given by the accelerometer, the velocity and the displacement of the projectile are also known. A Zimmer camera is used to give a second measure of the projectile displacement. For concrete beams, special supports have been designed that let the beam rotate but prevent any vertical rebound. Force sensors measure the reaction on the supports. Finally a digital high speed camera films the specimen or the beam during the impact. The acquisition frequency chosen goes from 3000 frames per second to 10000, which requires the use of a powerful external lighting. The complete set-up for impacts on beams is presented in figure 1.

Image correlation is efficient only if the recorded images present a wide enough gray level spectrum. The specimen used for dynamical brazilian tests are obtained by cutting up a larger specimen so that the structure of concrete is visible (aggregates, sand, cement and voids) and thus the images are suitable for images correlation. On the contrary, for reinforced concrete beams, we record the lateral face of the beam which is uniformly gray, therefore a random texture was applied onto this surface prior to the experiment to make the correlation possible by creating local gray level fluctuations.

\subsection{Caracterization tests, brazilian test in dynamics}

Several specimen are tested in order to illustrate the role that play the impact velocity and the specimen length. Two impact velocities are compared $V_{0}=2.42 \mathrm{~m} . \mathrm{s}^{-1}$ and $V_{0}=1.47 \mathrm{~m} . \mathrm{s}^{-1}$ and two length $l_{0}=22 \mathrm{~cm}$ and $l_{0}=$ $9.4 \mathrm{~cm}$. The results in terms of impact forces measured are presented in figure 2.

We can observe on these curves that the impact velocity has almost no influence on the maximum impact force measured, while it is directly proportional to the specimen length. The impact velocity plays a role on the value of the second load peak which increases with the velocity. It is the energy dissipated, which is proportional to the area under the impact force curve, which is affected by velocity.

The figure 3 presents the images obtained with the high speed camera during a brazilian test. The fragmentation 


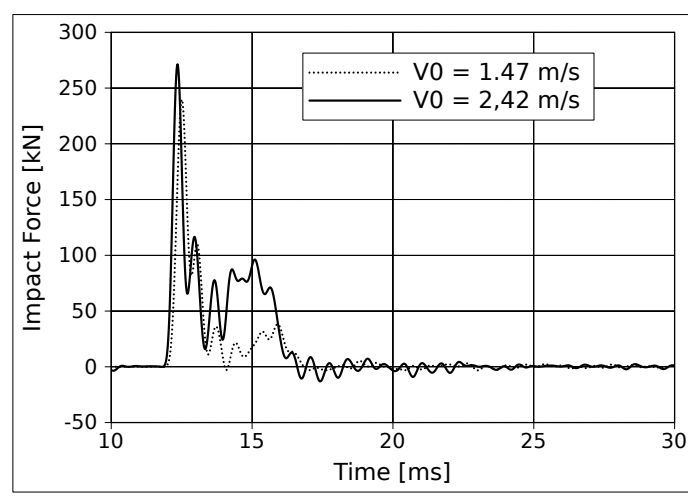

(a) Influence of impact velocity, $L_{0}=22 \mathrm{~cm}$

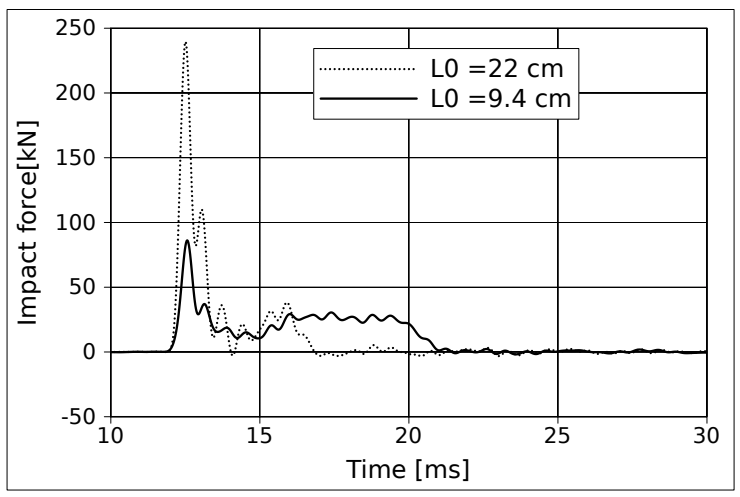

(b) Influence of specimen length, $V_{0}=2.42$ $\mathrm{m} . \mathrm{s}^{-1}$

Figure 2: Impact force measured for dynamic Brazilian tests

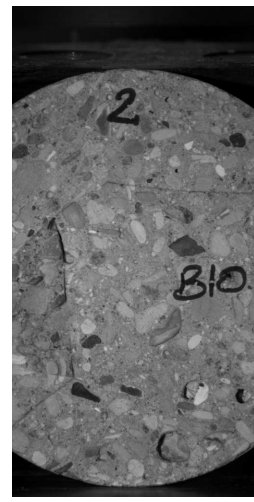

(a) Before impact

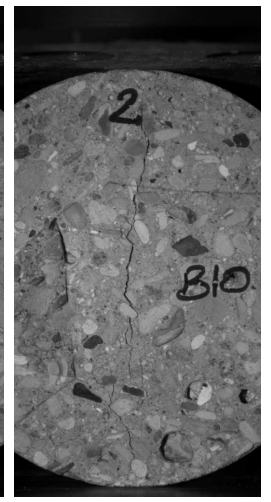

(b) $\mathrm{t}=0.166$ $\mathrm{ms}$

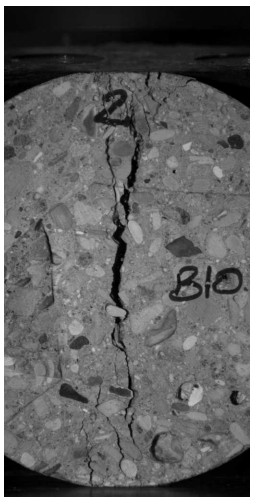

(c) $\mathrm{t}=1.0$ $\mathrm{ms}$

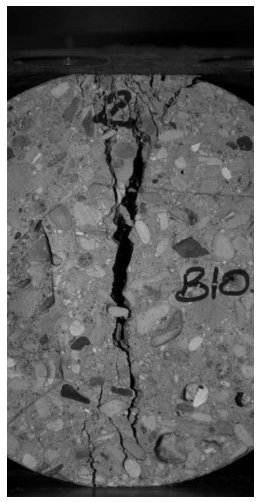

(d) $\mathrm{t}=1.833$

$\mathrm{ms}$

Figure 3: Cracking evolution during dynamic Brazilian test, $L_{0}=22 \mathrm{~cm}, V_{0}=1.42 \mathrm{~m} . \mathrm{s}^{-1}$.

process is the following: first $(\mathrm{t}=0.166 \mathrm{~ms}$, concrete is damaged in tension leading to a vertical crack starting from the upper part of the specimen (but not on the upper edge, loaded in compression). This crack then opens and propagates toward the bottom. At the same time $(\mathrm{t}=1.0 \mathrm{~ms})$, the region around edges start to damage, and we can observe multiple cracks creating several fragments. The damage is due to the compression loading state encountered in these zones. After that point, the specimen is completely broken, and the cracks only open more $(\mathrm{t}=1.833 \mathrm{~ms})$.

Figures 4(a) and 4(b) represent respectively the horizontal and vertical displacement fields obtained by images correlation (Besnard, Hild, and Roux 2006). The horizontal displacement field shows that the time that takes the main vertical crack to propagate is lower that the time between two recorded images (i.e. less than $0.166 \mathrm{~ms}$ ). For more interesting results, for instance to measure the crack velocity, it would be necessary to increase the acquisition speed, which cannot be done with the technology used for these tests.

\subsection{Impact on reinforced concrete beams}

Only three tests have been performed on reinforced concrete beams, with nevertheless the objective of getting to two different failure modes: a ductile failure by bending and a brittle failure with shear cone. In that purpose, two different lengths and two different reinforcements are compared. Figure 6 summarizes the differences between the different tests. The other parameters of the geometry and reinforcement can be found in figure 5 . We can notice in figure 5(a) that the longitudinal reinforcements are welded at the ends of the beam to prevent any sliding.

In figure 5(b), we can see that the lower reinforcing bars have a diameter of $20 \mathrm{~mm}$, the upper ones a $8 \mathrm{~mm}$ diameter and the stirrups a $6 \mathrm{~mm}$ one. The beams have a section of $150 * 200 \mathrm{~mm}^{2}$.

The failure facies are presented in figure 7 . For the bending failure mode, we can clearly see that the cracks 


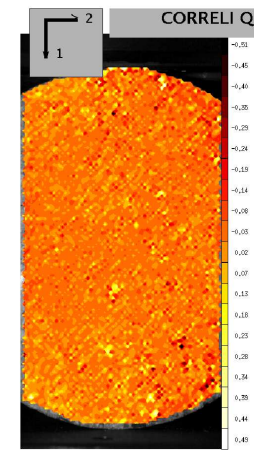

$\mathrm{t}=0 \mathrm{~ms}$

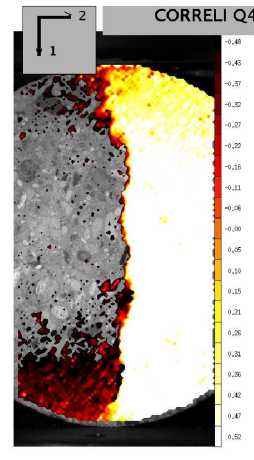

$\mathrm{t}=0,166 \mathrm{~ms}$

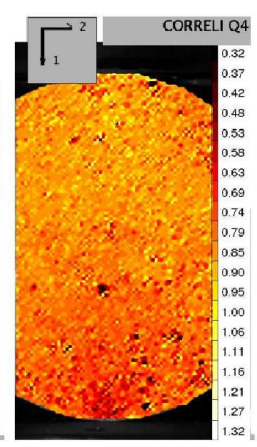

$\mathrm{t}=-0,166 \mathrm{~ms}$

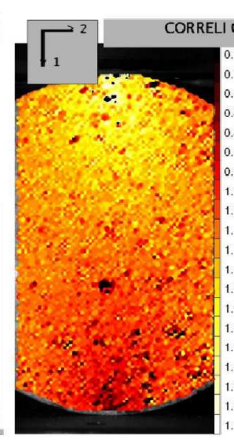

$\mathrm{t}=0 \mathrm{~ms}$

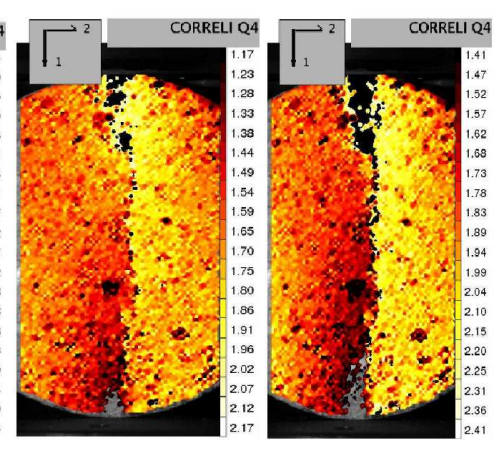

$\mathrm{t}=0,166 \mathrm{~ms}$

(a) Horizontal displacement

(b) Vertical displacement

Figure 4: Displacement field obtained by images correlation with CORRELI in pixels.

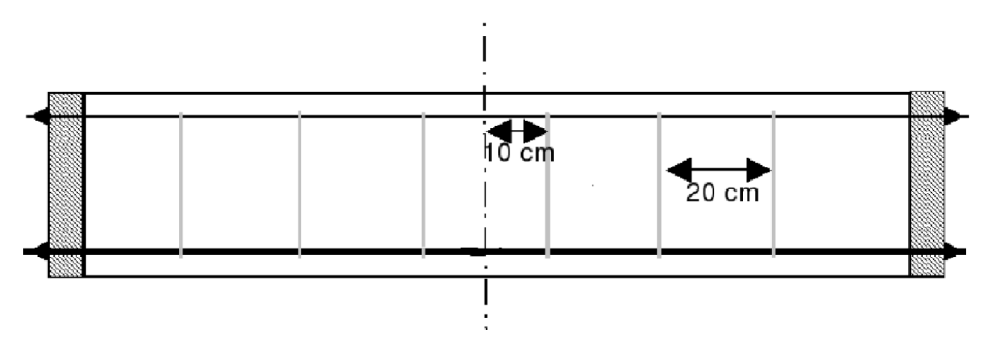

(a) Beams reinforcements description

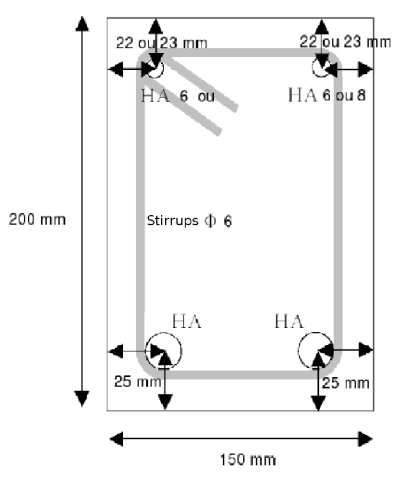

(b) Cross-section

Figure 5: Geometry and reinforcement of the reinforced concrete beams

\begin{tabular}{|c|c|c|c|}
\hline Beam & Length & Span & Stirrup \\
\hline P 1 & $1,8 \mathrm{~m}$ & $1,5 \mathrm{~m}$ & Yes \\
\hline P 2 & $1,3 \mathrm{~m}$ & $1,0 \mathrm{~m}$ & No \\
\hline P 3 & $1,3 \mathrm{~m}$ & $1,0 \mathrm{~m}$ & Yes \\
\hline
\end{tabular}

Figure 6: Design differences 


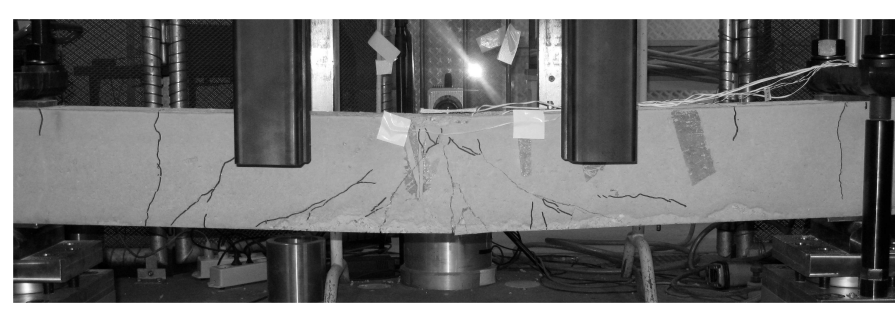

(a) Bending failure: Beams P1 and P3

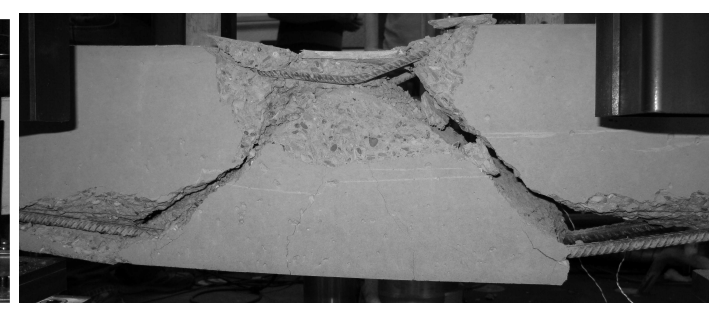

(b) Punching cone failure: Beam P2

Figure 7: Failure facies for the two failure modes obtained on a reinforced concrete beam under impact $\left(V_{0}=8.43 \mathrm{~m} / \mathrm{s}\right)$

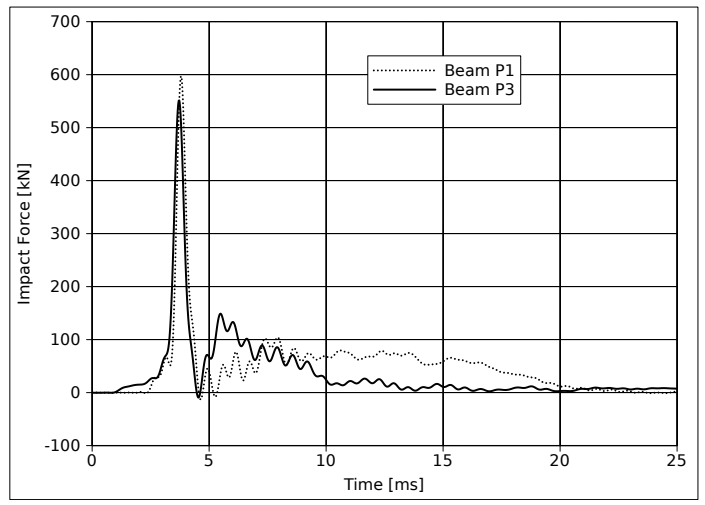

Figure 8: Comparison between impact forces measured for the bending failure (Beam P1) and the punching cone failure (beam P3).

on the bottom face are more inclined as long as we move away from the middle of the beam. We can also observe cracks on the upper face near supports due to the dynamical aspect of bending. For the short beam without stirrups, a concrete plug comes undone right under impact, only maintained by the longitudinal reinforcements.

The figure 8, that shows the impact forces measured for the two failure modes, exhibits a very small difference between the two curves. The maximal force measured is almost the same in both case, as the first impact duration. A significant difference is only observed in the second load peak.

\section{FINITE ELEMENTS ANALYSIS}

\subsection{Anisotropic delay-damage model for concrete in dynamics}

For concrete, the microcracks due to tension are mainly orthogonal to the loading direction, when the microcracks due to compression are mainly parallel to the loading direction. The damage state has then to be represented by a tensorial variable $D$ (either a fourth rank tensor or a second rank tensor) (Leckie and Onat 1981; Krajcinovic 1985). The use of a second order damage tensor is more convenient for practical applications (as well as for the material parameters identification) and this is the choice made here. The damage anisotropy induced by either tension or compression is then simply modeled by the consideration of damage evolution laws ensuring a damage rate proportional to the positive part of the strain tensor, i.e. a damage governed by the principal extensions (Mazars, Berthaud, and Ramtani 1990; Dragon and Halm 1998).

The basis of the present work is the rate-independant anisotropic damage model proposed by (Desmorat, Gatuingt, and Ragueneau 2007). According to the thermodynamics framework, the single damage variable $D$ is considered and a single set of material parameters is valid for tension and compression.

The full set of proposed constitutive equations including anisotropic delay-damage reads :

- elasticity

$$
\epsilon=\frac{1+\nu}{E} \tilde{\boldsymbol{\sigma}}-\frac{\nu}{E} \operatorname{tr} \tilde{\boldsymbol{\sigma}} \mathbf{1} \quad \text { or } \quad \boldsymbol{\epsilon}=\underline{E}^{-1}: \tilde{\boldsymbol{\sigma}}
$$

with $E$ the young's modulus, $\nu$ the Poisson's ration and $\underline{E}$ the Hooke's tensor.

- effective stress

$$
\tilde{\boldsymbol{\sigma}}=\left[(\mathbf{1}-\boldsymbol{D})^{-1 / 2} \boldsymbol{\sigma}^{D}(\mathbf{1}-\boldsymbol{D})^{-1 / 2}\right]^{D}+\frac{1}{3}\left[\frac{\langle\operatorname{tr} \boldsymbol{\sigma}\rangle_{+}}{1-\operatorname{tr} \boldsymbol{D}}+\langle\operatorname{tr} \boldsymbol{\sigma}\rangle_{-}\right] \mathbf{1}
$$




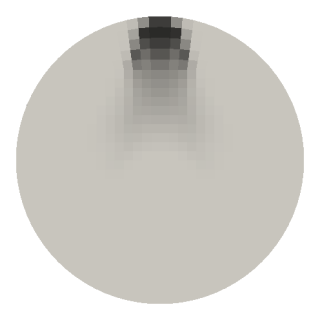

(a) $\mathrm{t}=0,05 \mathrm{~ms}$

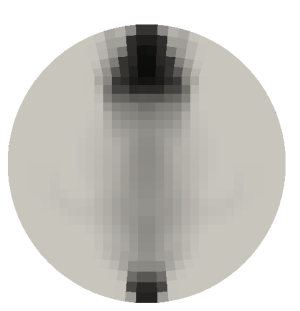

(b) $0,1 \mathrm{~ms}$

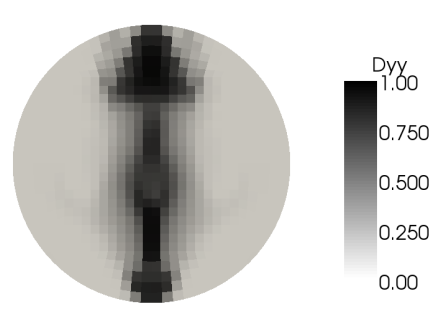

(c) $0,15 \mathrm{~ms}$

Figure 9: Damage $D_{y y}$ propagation

where $(\bullet)^{D}$ denotes the deviatoric part, $\langle\bullet\rangle_{+}\left(\right.$resp. $\left.\langle\bullet\rangle_{-}\right)$the positive (resp. negative) part of a scalar.

- Mazars damage criterion

$$
f=\kappa^{-1}(\hat{\epsilon})-\operatorname{tr} \boldsymbol{D}, \quad \hat{\epsilon}=\sqrt{\langle\boldsymbol{\epsilon}\rangle_{+}:\langle\boldsymbol{\epsilon}\rangle_{+}}=\sqrt{\operatorname{tr}\langle\boldsymbol{\epsilon}\rangle_{+}^{2}}
$$

using a viscous regularization, so that the condition $f \leq 0$ corresponds to elastic loading or unloading and the condition $f>0$ corresponds to damage growth. In this last case one writes then:

$$
f=-\frac{1}{b} \ln \left(\frac{\dot{D}_{\infty}-\operatorname{tr} \dot{\boldsymbol{D}}}{\dot{D}_{\infty}}\right)
$$

$\langle\epsilon\rangle_{+}$is the positive part of the strain tensor build from the positive eigen strains.

The material parameters $\dot{D}_{\infty}$ and $b$ are the delay-damage parameters and the $\kappa^{-1}$ function is set as

$$
\kappa^{-1}(\hat{\epsilon})=g(\hat{\epsilon})=a A\left[\arctan \left(\frac{\hat{\epsilon}}{a}\right)-\arctan \left(\frac{\kappa_{0}}{a}\right)\right]
$$

introducing $\kappa_{0}$ as damage threshold, $A$ and $a$ as damage parameters;

- induced damage anisotropy governed by the positive extensions,

$$
\dot{D}=\dot{\lambda}\langle\epsilon\rangle_{+}^{2}
$$

The damage multiplier $\dot{\lambda}$ is determined from the damage criterion expression for $f>0$ (Equation [4]).

The delay-damage law introduced by (Allix and Deü 1997) is recovered from previous equations and extended to induced anisotropy as:

$$
\operatorname{tr} \dot{\boldsymbol{D}}=\dot{D}_{\infty}[1-\exp (-b(g(\hat{\epsilon})-\operatorname{tr} \boldsymbol{D}))]
$$

At the final stage of the numerical implementation the elasticity law needs to be inverted. This can be done in a closed form as:

$$
\begin{aligned}
\boldsymbol{\sigma}= & (\mathbf{1}-\boldsymbol{D})^{1 / 2} \tilde{\boldsymbol{\sigma}}(\mathbf{1}-\boldsymbol{D})^{1 / 2}-\frac{(\mathbf{1}-\boldsymbol{D}): \tilde{\boldsymbol{\sigma}}}{3-\operatorname{tr} \boldsymbol{D}}(\mathbf{1}-\boldsymbol{D}) \\
& +\frac{1}{3}\left[(1-\operatorname{tr} \boldsymbol{D})\langle\operatorname{tr} \tilde{\boldsymbol{\sigma}}\rangle_{+}+\langle\operatorname{tr} \tilde{\boldsymbol{\sigma}}\rangle_{-}\right] \mathbf{1}
\end{aligned}
$$

Figure 10a shows the monotonic stress-strain curves for concrete in tension. Quasi-static and dynamic responses (at different strain rates) are plotted.The material parameters describing well concrete quasi-static behavior are: $E=42 \mathrm{GPa}, \nu=0.2, \kappa_{0}=510^{-5}, A=510^{3}, a=2.9310^{-4}$. The viscous regularization parameters are $b=1$ and $\dot{D}_{\infty}=50000 \mathrm{~s}^{-1}$. The rate effect obtain with these parameters are closed to those experimentally observed. Indeed using a modified version of the Hopkinson bar test, Klepaczko and Brara (Klepaczko and Brara 2001) obtained an increase of tensile resistance of 10 for a $100 \mathrm{~s}^{-1}$ strain rate. Figure $10 \mathrm{~b}$ shows different damage rate evolutions for $b \times D_{\infty}=50000$ constant. 

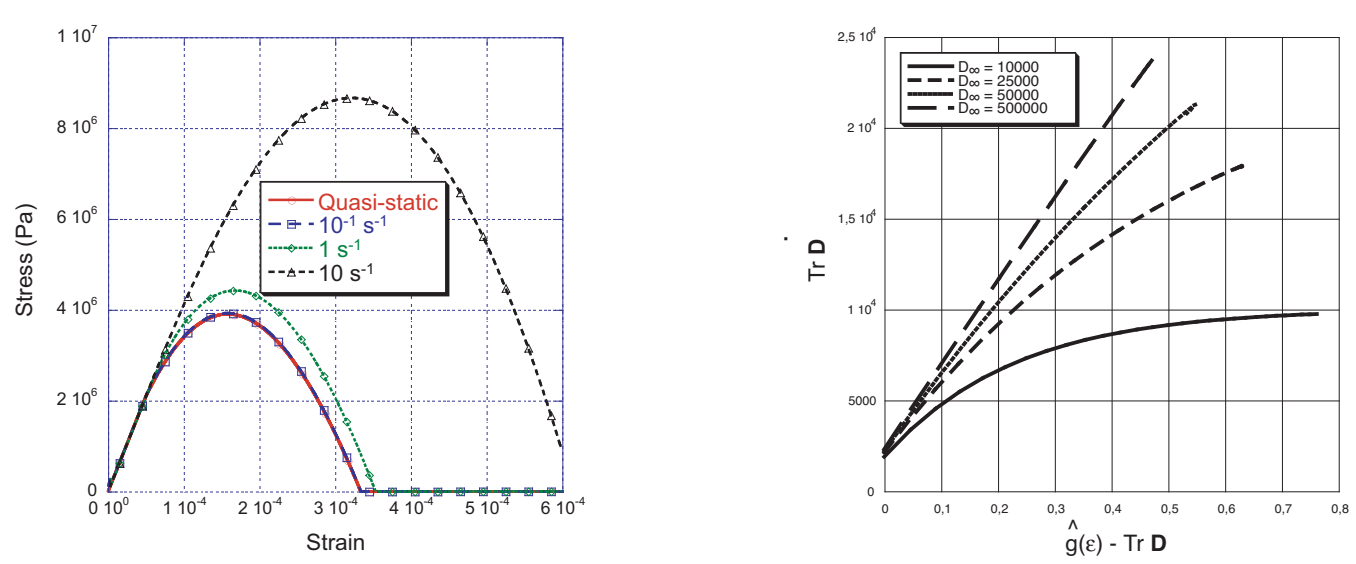

Figure 10: a/ Stress-strain curves for concrete at different loading rates, b/ Saturation of the damage rate for different $\dot{D}_{\infty}$

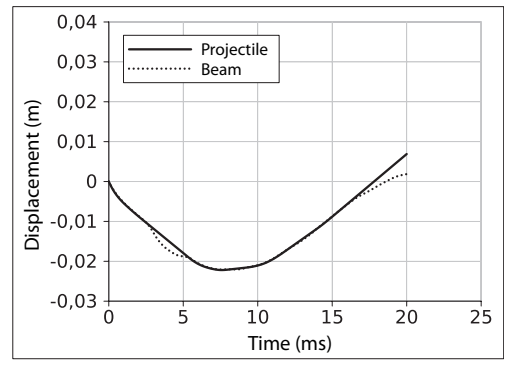

(a) Beam P1

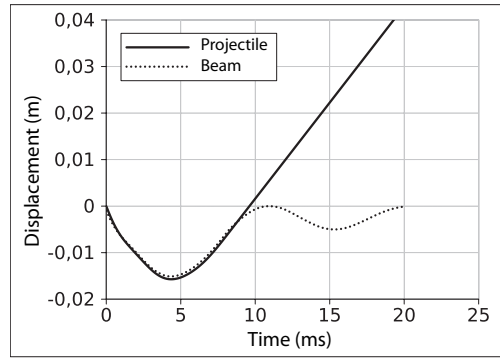

(b) Beam P3

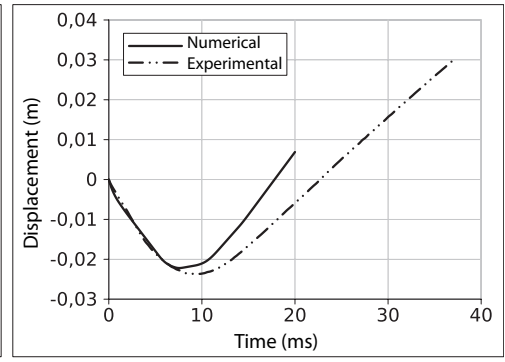

(c) Comparison with the test

Figure 11: Displacement vs time

\subsection{Brazilian dynamic tests}

The geometry and loading are those of the experiments presented section 2.2. The computer code used (EUROPLEXUS) allow to simulate the impact between two solids. It reproduces exactly the load by modeling the specimen and the projectile which we impose a speed equal to the impact velocity. The mesh is composed of 8 Gauss points cubic elements for the concrete specimen (7200 elements) and 6 Gauss points prismatic elements (900) for the projectile. For the three samples tested damage follows the same evolution. It first appears under the impact and to support. Then propagate two cracks which finally meet in the center of the specimen (figure 9). The crack propagation takes place in less than $0.05 \mathrm{~ms}$ that is in a period less than the acquisition frequency of the camera as experimentally observed.

\subsection{Beam impact tests}

This section is devoted to numerical modeling of impact tests on reinforced concrete beams. The numerical simulations correspond to tests on structures presented in section 2.3. The mesh of the beam consists of 5 cubic elements in width, 10 in height and 130 in length for the short beam (180 beam for the long one), which correspond to a total of 6500 elements (9000 for long). The reinforcement bars were modeled by bar type elements connected to the 3D mesh of the beam (no slinding). We used a perfect elastic plastic behavior for the bar.

Experimental and numerical impact forces were compared for beams P1 and P3. The results are good for the short beam P3 except for the first peak that is underestimated by the simulation. The duration of the first peak and above the second phase of impact, corresponding to the opening of the shear cone are fairly well reproduced. For the long beam P1, the simulation gives less satisfactory results. The first peak stress is much higher than the experimental response. Especially the post-peak part shows rebounds that are not observed during the test. Figure 11(a) and 11(b) compares for beams P1 and P3 displacement of the beam and the projectile. For P3, the beam and the projectile remain in contact until the rebound and then the beam begins to vibrate. For the 


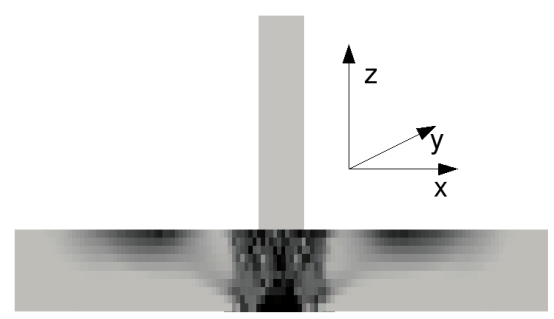

(a) $\mathrm{t}=0,25 \mathrm{~ms}$

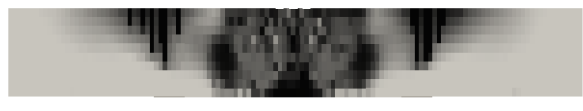

(b) $\mathrm{t}=0,50 \mathrm{~ms}$

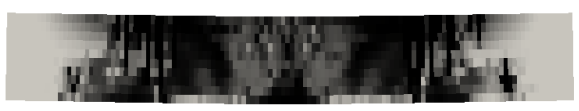

(c) $\mathrm{t}=1,50 \mathrm{~ms}$

Figure 12: Damage $D_{X X}$ map for the short beam P3

long beam (P1), the impact is longer, so that for the same simulation time, it does not appear a vibration phase. Comparison with experimental results (figure 11(c)) shows a good correlation for the beginning of the impact. However, the duration of impact calculated is shorter thus dissipating less which leads to a rebound speed more important than experimental one. Figure 12 shows the evolution of the damage computed during the impact.

\section{CONCLUSIONS}

In this work we developed experimental tests on drop-weight tower and an anisotropic damage model for concrete. These tests were instrumented to provide a database for validating the model developed. For the Brazilian tests, the impact force, acceleration and the displacement of the projectile were measured. A high speed camera was used to film the tests and analyze the degradation process. However, the acquisition speed of the camera used (6000 and 30000 images per second) is not fast enough to observe the cracks propagation. The Digital Image Correlation, however, allowed to observe the overall displacement of the beam and the specimen before cracking. The number of test is relatively low ( 4 Brazilian and 4 beams), so that few parameters have been studied.

The anisotropic model reproduces well (at least for the Brazilian test and impact test on beam) the cracking shape. Furthermore, numerical simulations have shown that this model allowed to carry the calculation to a stage where the total damage of the structure is very high. We can therefore characterize the model robust

\section{REFERENCES}

Allix, O. and J. Deü (1997). Delay-damage modelling for fracture prediction of laminated composites under dynamic loading. Engineering Transactions 45, 29-46.

Besnard, G., F. Hild, and S. Roux (2006). "Finite-Element” Displacement Fields Analysis from Digital Images: Application to Portevin-Le Châtelier Bands. Experimental Mechanics 46(6), 789-803.

Desmorat, R., F. Gatuingt, and F. Ragueneau (2007). Nonlocal anisotropic damage model and related computational aspects for quasi-brittle materials. Engineering Fracture Mechanics 74, 1539-1560.

Dragon, A. and D. Halm (1998). An anisotropic model of damage and frictional sliding for brittle materials. European Journal of Mechanics, A/Solids 17, 439-460.

Klepaczko, J. R. and A. Brara (2001). An experimental method for dynamic tensile testing of concrete by spalling. International Journal of Impact Engineering 25, 387-409.

Krajcinovic, D. (1985). Continuous damage mechanics revisited: basic concepts and definitions. J. Appl. Mech. 52(6), 829-834.

Leckie, F. A. and E. T. Onat (1981). Tensorial nature of damage measuring internal variables, Chapter Physical Non-Linearities in Structural Analysis, pp. 140-155. J. Hult and J. Lemaitre eds, Springer Berlin.

Mazars, J., Y. Berthaud, and S. Ramtani (1990). The unilateral behaviour of damaged concrete. Engineering Fracture Mechanics 35(4), 629-635.

Tedesco, J., C. Ross, and S. Kuennen (1993). Experimental and numerical analysis of high strain rate splitting tests. ACI Materials journal 90, 162-169. 